\title{
Use of Simulated Data for the Estimation of Prior Models in Kalman Filter-Based ECGI
}

\author{
Yesim Serinagaoglu Dogrusoz, Taha Erenler \\ Electrical and Electronics Engineering Department, \\ Middle East Technical University, Ankara, Turkey
}

\begin{abstract}
Kalman filtering has been successfully applied to electrocardiographic imaging (ECGI) to improve the estimation accuracy, especially when a 'good' training set of epicardial potentials is available to estimate the prior statistics. Most methods in the literature use previously measured experimental data to obtain these training sets, which would not be feasible in a clinical application. In this study we explored the effectiveness of using simulated epicardial potentials and the corresponding BSPS for obtaining the prior models based on two approaches: maximum likelihood (MLIF) and maximum a posteriori (MAPIF) estimation. Our results showed that even using a simple simulation method, and large margin in the initial pacing location range (80mm here), simulated data can be used for defining the prior models in the Kalman filterbased-ECGI.
\end{abstract}

\section{Introduction}

Electrocardiographic imaging (ECGI) aims to reconstruct the electrical activity of the heart based on multielectrode body surface potential (BSP) measurements and a mathematical model relating the sources to these measurements. This inverse problem is ill-posed; small disturbances in the BSPs yield large errors in the solutions. The remedy for this ill-posedness is to apply regularization.

Statistical estimation methods have been applied successfully for solving the inverse ECG problem [1-4]. These method have great flexibility to represent the data, and they provide performance evaluation tools for quantification of uncertainties and errors in the model. However, for an accurate inverse reconstruction, they require "good" a priori information. It has been a great challenge to obtain the said prior information, when we have limited knowledge about the sources themselves.

In our previous studies, we applied the Kalman filter to solve the inverse ECG problem. We used data from animal experiments to evaluate the statistical estimation meth- ods. In those studies, both the electrograms (EGM) and the BSPs were available, thus the $a$ priori Kalman filter parameters were estimated from training sets consisting of those measurements $[3,4]$. However, in a clinical application, such measurements are unavailable. In this study, we explore the use of simulated data (EGM and BSP) for constructing appropriate training sets, which then can be used to estimate the desired a priori parameters.

\section{Problem Definition}

In the Kalman filtering approach to the inverse ECG problem, the epicardial potentials are defined as the state variables. Then, the problem can be formulated in a linear state-space form as follows:

$$
\begin{aligned}
\mathbf{y}_{k} & =\mathbf{A} \mathbf{x}_{k}+\mathbf{v}_{k} \\
\mathbf{x}_{k+1} & =\mathbf{F x}_{k}+\mathbf{w}_{k}
\end{aligned}
$$

where $\mathbf{x}_{k} \in \mathbb{R}^{N}$ and $\mathbf{y}_{k} \in \mathbb{R}^{M}$ denote the epicardial and the body surface potential vectors at time $k$, respectively; $\mathbf{v}_{k} \in \mathbb{R}^{M} \sim \mathcal{N}\left(\mathbf{v}_{k} ; \mathbf{0}, \mathbf{R}\right)$ and $\mathbf{w}_{k} \in \mathbb{R}^{\mathbb{N}} \sim \mathcal{N}\left(\mathbf{w}_{k} ; \mathbf{0}, \mathbf{Q}\right)$ correspond to measurement and process noises, respectively; $\mathbf{F}$ is the $N \times N$ state transition matrix and $\mathbf{A}$ is the $M \times N$ forward transfer matrix. Furthermore, the process and measurement noises are assumed to be uncorrelated with the state $\mathbf{x}_{k}$.

Kalman filter is an optimal estimator used for reconstructing the unobserved states of a system by using the observed data. There are two stages in the Kalman filter. In the first stage, the predicted state vector $\mathbf{x}_{k \mid k-1}$ and the state covariance are estimated as:

$$
\begin{aligned}
\mathbf{x}_{k \mid k-1} & =\mathbf{F} \mathbf{x}_{k-1 \mid k-1} \\
\mathbf{P}_{k \mid k-1} & =\mathbf{F P}_{k-1 \mid k-1} \mathbf{F}^{T}+\mathbf{Q}
\end{aligned}
$$

Second stage is about updating the predictions $\mathbf{x}_{k \mid k-1}$ and $\mathbf{P}_{k \mid k-1}$ by using the measurement vector $\mathbf{y}_{k}$ to estimate the state vector, $\mathbf{x}_{k \mid k}$, and the state covariance matrix, $\mathbf{P}_{k \mid k}$ 
as follows:

$$
\begin{aligned}
\mathbf{K}_{k} & =\mathbf{P}_{k \mid k-1} \mathbf{A}^{T}\left(\mathbf{A} \mathbf{P}_{k \mid k-1} \mathbf{A}^{T}+\mathbf{R}\right)^{-1} \\
\mathbf{x}_{k \mid k} & =\mathbf{x}_{k \mid k-1}+\mathbf{K}_{k}\left(\mathbf{y}_{k}-\mathbf{A} \mathbf{x}_{k \mid k-1}\right) \\
\mathbf{P}_{k \mid k} & =\left(\mathbf{I}-\mathbf{K}_{k} \mathbf{A}\right) \mathbf{P}_{k \mid k-1}
\end{aligned}
$$

where $\mathbf{K}_{k}$ is the Kalman gain. In order to initialize the Kalman filter at the first time instant and run the filter recursively, the mean vector $\overline{\mathbf{x}}_{1}=\mathbf{x}_{0 \mid 0}$ and covariance matrix $\boldsymbol{\Sigma}_{1}=\mathbf{P}_{0 \mid 0}$, must be found. Furthermore, the state transition matrix $\mathbf{F}$, and the error covariances $\mathbf{R}$ and $\mathbf{Q}$ also must be specified.

This study utilizes ML and MAP techniques to estimate the unknown model parameter set $\Theta=$ $\left\{\overline{\mathbf{x}}_{1}, \boldsymbol{\Sigma}_{1}, \mathbf{F}, \mathbf{Q}, \mathbf{R}\right\}$. A database which consists of epicardial potentials $\mathbf{X}_{t r}=\left\{\mathbf{x}_{1: T}^{\ell}\right\}_{\ell=1: L}$ and corresponding body surface potentials $\mathbf{Y}_{t r}=\left\{\mathbf{y}_{1: T}^{\ell}\right\}_{\ell=1: L}$ are used by the ML- and MAP-based parameter estimation algorithms. Details of these methods can be found in [4].

\section{Test and Training Sets}

In this study we used data from experiments carried out at the University of Utah, Nora Eccles Harrison Cardiovascular Research and Training Institute (CVRTI) [5]. In these experiments, epicardial potentials were measured from perfused and ventricularly paced dog hearts, which were suspended in a human shaped electrolytic tank. Sampling rate was $1000 \mathrm{samp} / \mathrm{s}$, and the sock recording setup consisted of 490 nodes. The body surface potentials (BSP) were simulated from these measured epicardial potentials by multiplying them by a forward transfer matrix, which was calculated using the boundary element method (BEM) [6] and adding white Gaussian noise at $30 \mathrm{~dB}$ SNR.

We assigned one beat from a single dog experiment as the test beat for solving the inverse problem. We used two different approaches for constructing the training set for estimating the prior parameters:

- Measured TS: The electrograms measured in experiments different from the test beat and the corresponding BSPs were used to construct the first training set.

- Simulated TS: We used simulated electrograms and the corresponding BSPs for this second training set. First the 3D transmembrane potentials (TMPs) were simulated in an anisotropic heart using the Aliev-Panfilov model [7]. In these computations, the same set of simulation parameters were used, neglecting differences in the electrophysiology of different types of heart cells. The Auckland experimental heart model with 264477 myocardial nodes was used for this computation [8]. Then, the corresponding epicardial potentials were obtained by solving the forward problem using the bidomain model and the finite element method. Finally, the simulated EGMs were mapped onto the Utah heart geometry using registration.

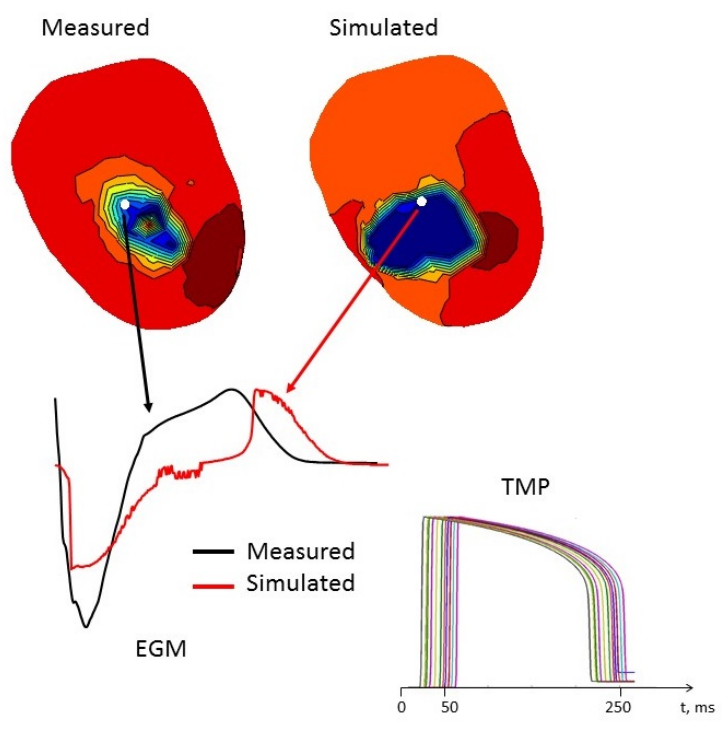

Figure 1. Measured and simulated epicardial potential data examples; isopotential maps and sample EGMs for each data from the same node are depicted. TMPs for several myocardial nodes were also illustrated.

The training set beats were paced from an $80 \mathrm{~mm}$ neighborhood of the pacing location of the test beat.

Figure 1 shows measured and simulated data examples, paced from the same epicardial node. Isopotential maps as well as electrograms for the same epicardial node (indicated as a white dot on the heart) are presented. TMPs from several nodes, which have similar morphology for all heart nodes, were also included.

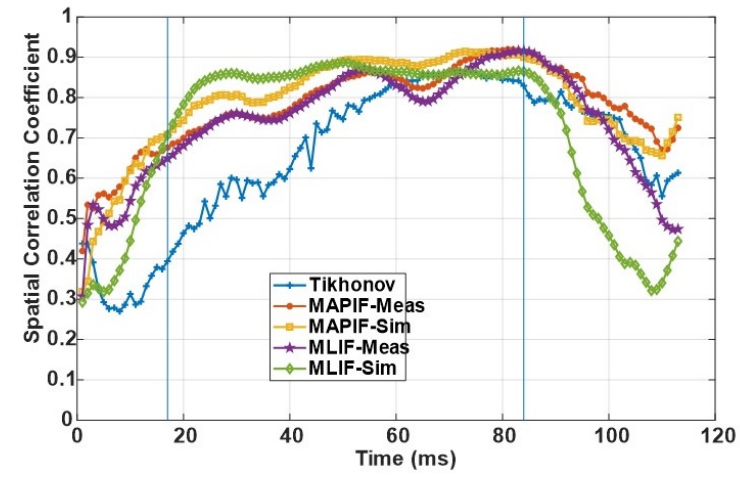

Figure 2. Spatial CC value across time for all methods. The vertical lines indicate the time instances for which the epicardial maps are shown in figure 3 . 
A
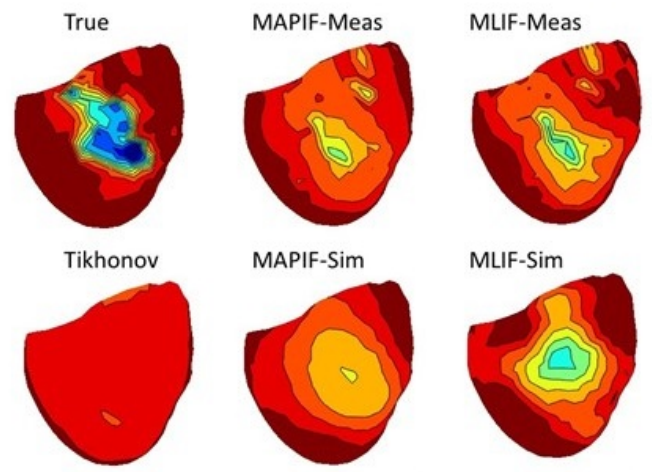

$\mathrm{t}=17 \mathrm{~ms}$
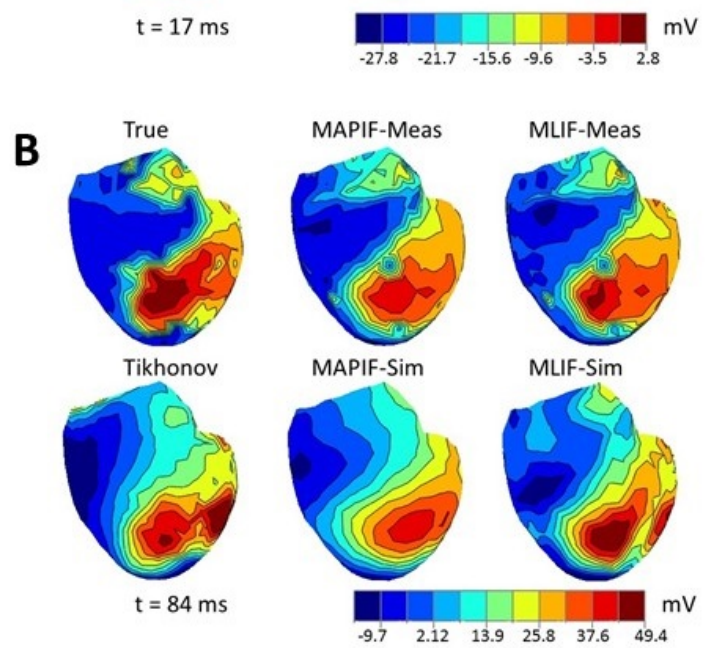

Figure 3. Sample epicardial maps at two different time instants; $t=17 \mathrm{~ms}$ in panel $\mathrm{A}$, and $t=84 \mathrm{~ms}$ in panel $\mathrm{B}$.

\section{Results}

Reconstructed electrograms were evaluated using the spatial (sCC) and temporal (tCC) correlation coefficients; the activation times (ATs) were computed using a spatiotemporal approach [9] and evaluated using Pearson's CC; pacing site localization accuracy was assessed based on the localization error (LE).

Figure 2 shows the spatial $\mathrm{CC}$ values as a function of time for all reconstructed EGM maps. The performance of the Kalman filter-based methods outperformed Tikhonov regularization for the first $60 \mathrm{~ms}$ of the activity. Throughout most of the QRS region, both MAPIF and MLIF using the simulated TS had similar, at some instances even higher sCC values compared to using the measured TS. After about $90 \mathrm{~ms}$, which is close to the end of the QRS region, the performance of MLIF with the simulated TS started to degrade.

Epicardial maps at two different time instants are given in figure 3. These time instants are representatives from an earlier time in the QRS (panel A, $17 \mathrm{~ms}$ ) and a later time in the QRS (panel B, $84 \mathrm{~ms}$ ), as marked by vertical lines

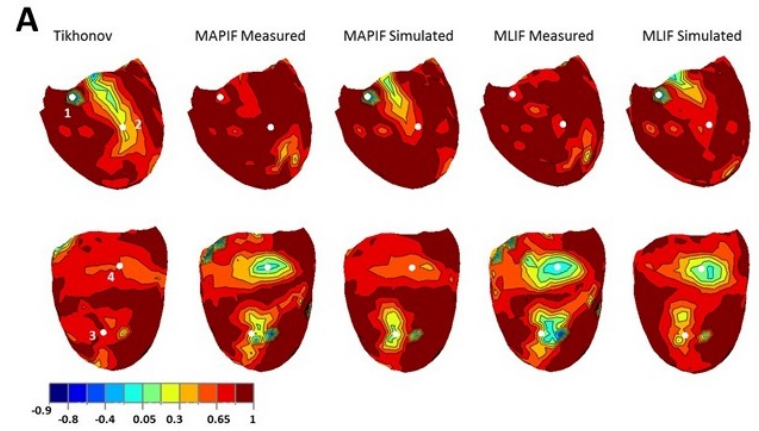

B
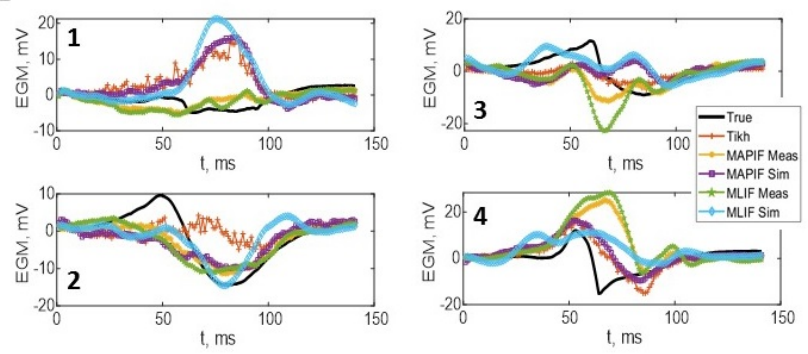

Figure 4. Panel A: Temporal CC values over the heart surface from the LV and RV views of the heart. Four nodes are markes as white dots corresponding to low tCC regions on these maps. Panel B: Electrograms corresponding to the marked low tCC nodes.

on the spatial CC plots of figure 2. Supporting the spatial $\mathrm{CC}$ results, at $17 \mathrm{~ms}$, all Kalman filter results outperformed Tikhonov regularization. At $84 \mathrm{~ms}$, all maps had similar distributions. Reconstructions using the simulated TS had good fidelity to the original map; MLIF especially had a comparable performance to the methods using the measured TS. MAPIF with the simulated TS, on the other hand, slightly smeared the wavefront.

Figure 4 shows the temporal $\mathrm{CC}$ values mapped onto the heart surface (panel A) from two different views; top and bottom rows correspond to the left (LV) and right (RV) ventricles, respectively. Both MAPIF and MLIF using the simulated TS had similar, at some nodes even higher tCC values compared to using the measured TS. In the LV-view, all Kalman filter methods outperformed the Tikhonov regularization. However, in the RV-view, there were small regions with small and/or negative tCC values corresponding to the same methods. Among the four Kalman filter-based results, MAPIF with the simulated TS only dis not have a low-tCC region around node 4 . Two nodes from each view were selected from low tCC regions, and the corresponding EGMs were also plotted (panel B). The EGMs shown here were representatives of the worst performances of the methods; the remaining EGMs closely resembled the measured potentials, as indicated by the high tCC values.

The quantitative metrics are summarized in table 1. 
Table 1. Summary of quantitative metrics evaluated in this study. These metrics are spatial CC, temporal $\mathrm{CC}, \mathrm{CC}$ of the ATs and LE (in mm). For sCC and tCC, the mean and standard deviation values are listed.

\begin{tabular}{|l|c|c|c|}
\hline & Method & Measured TS & Simulated TS \\
\hline \hline \multirow{3}{*}{ SCC } & MAPIF & $0.79 \pm 0.10$ & $0.80 \pm 0.12$ \\
\cline { 2 - 4 } & MLIF & $0.74 \pm 0.13$ & $0.72 \pm 0.19$ \\
\cline { 2 - 4 } & Tikhonov & \multicolumn{2}{|c|}{$0.67 \pm 0.16$} \\
\hline \multirow{3}{*}{ tCC } & MAPIF & $0.79 \pm 0.25$ & $0.82 \pm 0.20$ \\
\cline { 2 - 4 } & MLIF & $0.76 \pm 0.27$ & $0.77 \pm 0.22$ \\
\cline { 2 - 4 } & Tikhonov & \multicolumn{2}{|c|}{$0.79 \pm 0.20$} \\
\hline \multirow{3}{*}{ AT-CC } & MAPIF & 0.99 & 0.99 \\
\cline { 2 - 4 } & MLIF & 0.99 & 0.99 \\
\cline { 2 - 4 } & Tikhonov & \multicolumn{2}{|c|}{0.99} \\
\hline \multirow{3}{*}{ LE (mm) } & MAPIF & 8.94 & 6.36 \\
\cline { 2 - 4 } & MLIF & 8.94 & 4.46 \\
\cline { 2 - 4 } & Tikhonov & \multicolumn{2}{|c|}{8.94} \\
\hline
\end{tabular}

High spatial and temporal CC values of the Kalman filterbased methods using the simulated TS are also evident in these average values. ATs for all methods closely matched the true ATs, with $0.99 \mathrm{CC}$ value. $\mathrm{LE}$ values with the simulated TS-based method were the lowest.

\section{Discussion and Future Work}

In this study, we used simulated data as training sets to estimate the Kalman filter parameters. In these simulations, we did not take into account the heterogeneity of the TMPs for different types of heart cells. Instead, we used a simple generic TMP waveshape resulting from global Aliev-Panfilov simulation parameters.

Our results showed that, even using a simple simulation method, and large margin in the initial pacing location range $(80 \mathrm{~mm}$ here), simulated data can be used for defining the prior models in the Kalman filter-based-ECGI.

However, this study included only a single test case, and the BSPs were simulated rather than recorded simultaneously with the EGMs. This method needs further validation using more datasets, and especially measured BSPs.

\section{Acknowledgments}

This work was supported by The Scientific and Technological Research Council of Turkey, grant number 118E244. The authors would like to thank Dr. Robert S. MacLeod and his colleagues from University of Utah, Nora Eccles Harrison Cardiovascular Research and Training Institute for the data used in this study.

\section{References}

[1] Van Oosterom A. (1999) The use of the spatial covariance in computing pericardial potentials. IEEE Transactions on Biomedical Engineering, 46(7), 778-787.

[2] Serinagaoglu, Y., Brooks, D. H., MacLeod, R. S. (2006) Improved performance of Bayesian solutions for inverse electrocardiography using multiple information sources. IEEE Transactions on Biomedical Engineering, 53(10), 2024-2034.

[3] Aydin U., Serinagaoglu Dogrusoz, Y. (2011) A Kalman filter-based approach to reduce the effects of geometric errors and the measurement noise in the inverse ECG problem. Medical \& Biological Engineering \& Computing, 49(9), 1003-1013.

[4] Erenler, T., Serinagaoglu Dogrusoz, Y. (2019) ML and MAP estimation of parameters for the Kalman filter and smoother applied to electrocardiographic imaging. Medical \& Biological Engineering \& Computing, 57(10), 2093-2113.

[5] MacLeod R. S., Taccardi B., Lux R. L. (1995) Electrocardiographic mapping in a realistic torso tank preparation. In: Proceedings of 17 th International Conference of the EMBS, 45-246.

[6] Stanley P. C., Pilkington T. C., Morrow M N. (1986) The effects of thoracic inhomogeneities on the relationship between epicardial and torso potentials. IEEE Transactions on Biomedical Engineering, BME-33(3), 273-284.

[7] Aliev R. R., Panfilov A. V. (1996) A simple two-variable model of cardiac excitation. Chaos, Solitons \& Fractals, 7(3), 293-301.

[8] Nielsen P., Le Grice I., Smaill B., Hunter P. (1991) Mathematical model of geometry and fibrous structure of the heart. Am. J. Physiol, 260(29), H1365-H1378.

[9] Erem B., Coll-Font J., Orellana R. M., Št'oviček P., Brooks D. H. (2014) Using transmural regularization and dynamic modeling for noninvasive cardiac potential imaging of endocardial pacing with imprecise thoracic geometry. IEEE Transactions on Medical Imaging, 33(3), 726-738.

Address for correspondence:

Yesim Seringaoglu Dogrusoz, PhD.

Electrical-Electronics Engineering Department

Middle East Technical University, Ankara, Turkey

yserin@metu.edu.tr 